\title{
Sleep Problems in Children with Fetal Alcohol Spectrum Disorders
}

\author{
Maida Lynn Chen, M.D. '; Heather Carmichael Olson, Ph.D. ${ }^{2}$; Joseph F. Picciano, M.S. ${ }^{3}$; Jacqueline R. Starr, Ph.D. 4; Judith Owens, M.D., M.P.H. ${ }^{5}$
}

${ }^{1}$ Department of Pediatrics, Division of Pulmonary and Sleep Medicine, University of Washington School of Medicine, Seattle, WA; ${ }^{2}$ Department of Psychiatry and Behavioral Sciences, Division of Child Psychiatry, University of Washington School of Medicine, Seattle Children's Hospital Research Institute, Seattle, WA; ${ }^{3}$ Seattle Children's Hospital Research Institute, Center on Child Health, Development and Behavior, Seattle, WA; ${ }^{4}$ The Forsyth Institute, Cambridge, Massachusetts and University of Washington School of Public Health, Seattle, WA; 'Division of Pulmonary and Sleep Medicine, Children's National Medical Center, Washington, DC

Study Objectives: Sleep problems in children with fetal alcohol spectrum disorders (FASD) are reportedly common but not well characterized. Objectives were to: (1) assess sleep concerns in children with FASD using a caregiver-report survey, the Children's Sleep Habits Questionnaire (CSHQ); (2) compare CSHQ results with those of previously reported community sample; and (3) describe pilot polysomnography findings in children with FASD.

Methods: Children with FASD were recruited from a behavioral intervention study, and participating caregivers completed the CSHQ. CSHQ results were compared with the original data from a previously published community sample of similar age. Participants with FASD and elevated CSHQ scores were offered overnight polysomnography.

Results: Thirty-three children with FASD (4.1-12.1 years) were enrolled; $85 \%$ of children with FASD scored above the clinical cutoff Total Score of 41, reflecting marked sleep disturbance. Elevated subdomain scores occurred primarily in areas con- cerning for pediatric insomnia. Those with comorbid ADHD had elevated CSHQ on additional subdomains with no difference in Total Scores. Compared with the community sample, children with FASD had higher Total Scores on the CSHQ (52 vs. 39, $p<0.001$ ). Polysomnography, completed in 5 subjects, revealed mild sleep disordered breathing and fragmented sleep with elevated non-respiratory arousal indices.

Conclusions: Clinically significant sleep problems are present in children with FASD on both subjective and objective measures. Further investigation is needed to better describe these sleep disturbances and their impact on overall health and daytime neurobehavioral problems in this clinical population.

Keywords: sleep disorders; fetal alcohol spectrum disorders; prenatal alcohol; pediatric insomnia; school-age children; fetal alcohol syndrome; maternal pregnancy drinking

Citation: Chen ML; Olson HC; Picciano JF; Starr JR; Owens $\mathrm{J}$. Sleep problems in children with fetal alcohol spectrum disorders. J Clin Sleep Med 2012;8(4):421-429.
Cleep problems are reported to be common among children $\checkmark$ with fetal alcohol spectrum disorders (FASD), yet these sleep problems have not been well described in epidemiological studies..$^{1-3}$ FASD are characterized by lifelong neurodevelopmental disabilities arising from maternal consumption of alcohol during pregnancy., ${ }^{4,5}$ Recent data suggest that FASD can be identified in approximately $2 \%$ to $5 \%$ of younger school children in the U.S., making FASD a common public health problem. ${ }^{6}$ Individuals with FASD experience increased prevalence of problems with attention deficits, hyperactivity, and psychiatric conditions, ${ }^{1-8}$ leading to compromised daily function, ${ }^{8}$ caregiver and family function, ${ }^{9}$ and incurring high societal costs. ${ }^{10}$

Sleep integrity is crucial to the developmental process in children, and data suggest that disrupted sleep is associated with a wide range of health and behavioral problems in typically developing children, including poor school performance, neurocognitive deficits, daytime hyperactivity and inattention, mood instability, decreased growth, metabolic alterations, and cardiovascular sequelae. ${ }^{11-13}$ Treatment of the underlying sleep disorder leads to improvement or resolution of the clinical problem, suggesting a causal role. ${ }^{13,14}$

Importantly, this association is not limited to typically developing children. Multiple studies have found that both the

\section{BRIEF SUMMARY}

Current Knowledge/Study Rationale: Sleep problems in children with FASD, a common condition, are reportedly prevalent and clinically problematic, but not well described. Systematic characterization of these sleep problems is needed to better approach assessment and treatment of sleep disturbances in this challenging population.

Study Impact: Given findings of highly prevalent pediatric insomnia, and pilot findings of mild sleep disordered breathing and sleep fragmentation, care of children with FASD (who may diagnostically overlap with non-teratogenic ADHD) should include a clinical sleep health assessment and low threshold for polysomnography. Identification and treatment of sleep problems in those with FASD may lead to improvements in problematic daytime function.

prevalence and severity of sleep problems in children with underlying neurodevelopmental disorders are significantly higher, and appear to have a profound impact on daytime functioning of the child and family. In autism spectrum disorders, children characterized as "poor sleepers" have worsened scores on subjective caregiver questionnaires related to pediatric insomnia, supported by altered sleep architecture on formal sleep study. ${ }^{15}$ In attention deficit hyperactivity disorder (ADHD) which is a common comorbid diagnosis in those with FASD, ${ }^{16}$ sleep problems have been widely documented, including elevated rates of 
insomnia, periodic limb movement disorder, sleep disordered breathing, and increased daytime sleepiness on multiple sleep latency tests compared to controls. ${ }^{17}$ Improvement or resolution of ADHD symptoms with treatment of insomnia or other underlying sleep disorder has been reported..$^{13,18}$

Despite the demonstrated link between sleep problems and daytime difficulties in typically developing and other clinical populations, few studies have directly addressed sleep problems in those with FASD. ${ }^{19}$ Only one study has been published performing multidimensional assessment of sleep and daytime problems in FASD using standardized measures. Indeed, higher rates of both sleep and sensory problems were found in those with FASD compared to controls in that study. ${ }^{19}$ However, these findings need to be replicated in other cohorts. Many of the health, behavioral, and neurocognitive manifestations of pediatric sleep disturbance are conditions that are also known to be common problems among those with FASD. ${ }^{1,3,7}$ If a connection between sleep problems and daytime function can be established in FASD through further studies, proven effective treatments for these sleep disorders could be implemented in children with FASD. Such treatments could provide lifelong benefit for children with FASD, including reduction of health care and societal costs. ${ }^{20}$

The primary goal of this research was to begin building a comprehensive profile of sleep problems in this remarkably prevalent, but under recognized clinical population of children with FASD. Thus, study aims were as follows: (1) to systematically describe parent-reported sleep habits in a representative group of children with FASD by using the Children's Sleep Habits Questionnaire (CSHQ); (2) to compare these results to CSHQ results from a previously described community sample ${ }^{21}$; and (3) to obtain pilot and feasibility data from polysomnography (PSG) sleep assessment within a subgroup of the children with FASD. The authors hypothesized that children with FASD would show a higher rate of clinically concerning sleep problems on the CSHQ, compared with profiles of a typically developing community sample.

\section{METHODS}

\section{Participants}

\section{Children with FASD}

Thirty-three children were recruited from 34 participants enrolled in a behavioral intervention research study or related intervention training study in 2007. All participants enrolled in this subject pool were diagnosed with conditions within the umbrella category of FASD. All 34 families agreed to be enrolled, but one participant did not complete a sleep questionnaire and was therefore ineligible for the current descriptive study. All children in the subject pool were seen by an interdisciplinary team within the Washington State Fetal Alcohol Syndrome Diagnostic and Prevention Network (FAS DPN), and received a formal diagnosis on the fetal alcohol spectrum. Of the 33 participants, $88 \%$ had clinically significant externalizing behavior or attention problems on the Child Behavior Checklist (CBCL), which are common difficulties among children with FASD. ${ }^{7}$ Eligibility for the current study included a diagnosis on the fetal alcohol spectrum with confirmed alcohol exposure based on the clinically reproducible 4-Digit Diagnostic Code ${ }^{4}$ (see below); registration in a clinical database of a statewide network of FASD diagnostic clinics; residence in a designated geographic catchment area in Washington State; aged 4 to 12 years at time of enrollment; and residence with the current caregiver for $\geq 4$ months at enrollment with caregiver intent of maintaining that placement for at least the next 1.5 years to maximize retention in the larger behavioral intervention study.

Exclusion criteria for the larger study included other birth defect syndromes; known seizure disorder requiring daily medications; IQ $<55$; diagnosed thought disorder; very serious conduct problems (e.g., fire-setting); and current primary caregivers with alcohol abuse. Potential participants for both studies were not recruited based on any pre-study sleep assessments. Research was approved by the Institutional Review Boards (IRB) of the Seattle Children's Research Institute and Washington State Department of Social and Health Services, with further approval for comparison with de-identified community control data.

The 4-Digit Code is a standardized method for characterizing conditions across the fetal alcohol spectrum by using 4-point Likert scales to characterize 4 primary categories of definitional criteria. ${ }^{4}$ Higher scores indicate features in each category that are more similar to their classic representation in full FAS. These 4 categories include: (1) growth deficiency; (2) characteristic facial features; (3) central nervous system damage or dysfunction; and (4) level of prenatal alcohol exposure. Code combinations are collected into broader diagnostic categories, such as FAS or partial FAS. Non-FAS conditions seen in this study on the wider spectrum are grouped together as "static encephalopathy, alcohol-exposed" and "neurobehavioral disorder, alcohol-exposed" which other diagnostic systems may categorize as "alcohol-related neurodevelopmental disorders."

\section{Representativeness of Sample with FASD}

Because participants with FASD were recruited from a behavioral intervention study, care was taken to ascertain whether this sample of participants reflected the larger FASD population. Demographics, behavior profiles on the CBCL, comorbid ADHD diagnoses and diagnoses on the fetal alcohol spectrum were compared between this sample and the much larger Washington State FASD clinical database.

\section{Community Sample}

As part of a separate, already concluded study, comparison data were drawn from a large community sample $(n=418)$ of typically developing children, aged 4-11 years, enrolled in any of 3 public elementary schools during the 1997-98 academic year. ${ }^{21}$ Sample characteristics and study methods for this community sample have been fully reported elsewhere, ${ }^{21}$ though original data on individuals were used for this study's analyses. The community sample study was conducted to validate the CSHQ, so data were used in the current study only for descriptive comparison. The original community sample research project was approved by the IRB of Lifespan Hospitals, with further approval for current comparison to participants with FASD. 


\section{Measures}

\section{Demographic Data}

Demographic information included children's age, caregiver report of race/ethnicity, and gender. Family characteristics included measures of socioeconomic status (SES) based upon the Hollingshead Index ${ }^{22}$ and family structure (e.g., whether there was a father in household). Information on chronic medical conditions and medications was obtained via parent report as medical records were not consistently available. For participants with FASD, diagnostic category on the fetal alcohol spectrum was ascertained from clinical records.

\section{Sleep Habits}

Children's sleep habits were subjectively assessed by using the CSHQ, a validated 33-item caregiver questionnaire. The CSHQ includes key sleep domains grouped into 8 subscales: (1) Bedtime Resistance; (2) Sleep Onset Delay; (3) Sleep Duration; (4) Sleep Anxiety; (5) Night Wakings; (6) Parasomnias; (7) Sleep Disordered Breathing; and (8) Daytime Sleepiness. Most CSHQ items are rated on a 3-point scale ranging from "usually" (5 to 7 times/week) to "sometimes" (2 to 4 times/week) to "rarely" (0 to 1 time/week). Two questions about daytime sleepiness are rated on a 3-point scale of "not sleepy," "very sleepy," or "falls asleep." Excluding 2 items that were included in multiple subscales, the subscale inter-correlations for the community control group were $r=0.19$. The Total Score sums all 33 items, and higher values indicate more disturbed sleep. A Total Score $\geq 41$ has a sensitivity of $80 \%$ and specificity of $72 \%$ for differentiating between groups of children with and without sleep disorders diagnosed by a sleep medicine specialist. ${ }^{21}$

\section{Polysomnography}

Referral for a sleep medicine consultation and PSG was offered to participants with FASD whose CSHQ scores exceeded the clinical cutoff $(\geq 41)$. Consent was obtained to review medical records, including the sleep evaluation and PSG findings. Sleep medicine consultation and PSG were arranged by families (not study personnel), and not all families chose to pursue this evaluation.

Each PSG lasted $\geq 6 \mathrm{~h}$ and was performed in an accredited pediatric-specific sleep laboratory setting, which included a private darkened room free of distraction with a parent or guardian present. The following physiologic parameters were monitored: electroencephalogram, electro-oculogram, submental and anterior tibialis electromyograms, electrocardiogram, oronasal airflow measured by thermistor and pressure transducer, expired end-tidal carbon dioxide, oxygen saturation via pulse oximeter, and thoracic and abdominal movement. All data were recorded into a computer-based acquisition and analysis program (Rembrandt, Buffalo, NY), scored by one certified technician, and interpreted by a board certified sleep medicine physician in accordance with American Academy of Sleep Medicine (AASM) guidelines. ${ }^{23}$

Sleep architecture, including sleep stages, cortical arousals, awakenings, limb movements, and arousal indices were assessed by standard techniques. ${ }^{23}$ Respiratory disturbances were defined as follows: (1) Obstructive apnea: $\geq 80 \%$ reduction in airflow for 2 respiratory cycles in the presence of continued thoracic/abdominal effort; (2) Hypopnea: 30\% to $79 \%$ reduction in airflow for 2 respiratory cycles, accompanied by an EEG cortical arousal, awakening, and/or $\geq 3 \%$ oxygen desaturation; (3) Central apnea: $\geq 80 \%$ reduction in airflow with no respiratory effort, lasting $\geq 2$ respiratory cycles, and accompanied by an EEG arousal, awakening, and/or $\geq 3 \%$ oxygen desaturation. The apnea hypopnea index (AHI) was defined as the total number of respiratory disturbances averaged per hour of total sleep time, and further divided into those caused by obstructive apneas/hypopneas and those caused by central apneas.

Both attendant technician and interpreting physician were blinded to the CSHQ score and any involvement in an FASD intervention study, but given the clinical setting and behavioral considerations of the participants, could not be blinded to an underlying diagnosis of FASD in order to optimize patient safety and likelihood of successful data acquisition.

\section{Data Analysis}

For this exploratory study, descriptive statistics are reported using mean \pm standard deviation (SD) for normally distributed parameters or median with interquartile range (IQR) for those with skewed distributions. Because the fundamental aims focused on the CSHQ scores, formal comparisons were run only on CSHQ scores between the study and community sample groups for which raw data were available. Comparisons were carried out by using unpaired $t$-tests with 2 -sided p-values and significance set at $p<0.05$. Correction for multiple comparisons was not used in this exploratory study. Age, gender, SES, family structure, and presence of ADHD or other medical problems were found to vary by participant group, but none were found to correlate with CSHQ Total Score, so statistical adjustments for these factors were not performed in this pilot study. Preliminary evaluation for associations between and within CSHQ scores and markers of FASD severity was accomplished by using linear regression. All statistical analyses were performed using Stata, version 10 (College Station, TX).

For descriptive purposes, the group with FASD was further divided into those with and without caregiver report of ADHD for further descriptive comparison. Group characteristics of the community sample and larger Washington State FASD sample are provided for the reader's reference but were not statistically compared with this study's participants, given methodologic differences in original data collection.

Given the small number of children with PSG data, we report PSG parameters only as descriptive statistics, with published normative data from 388 typically developing children with a mean age of $6.8 \pm 0.48$ years (range 6.0-8.6 years) provided for reference. ${ }^{24}$

\section{RESULTS}

\section{Sample Characteristics}

\section{Demographics}

Within the group with FASD, mean age was $7.5 \pm 2.2$ years, with a slight predominance of males (Table 1). Fifty-eight percent were in adoptive families and $12 \%$ in foster families, while $18 \%$ of participants were living with one or both birth parents. 
Table 1-Group demographics and medical problems

\begin{tabular}{|c|c|c|}
\hline & FASD $(n=33)$ & Community $(n=418)$ \\
\hline Age, years, mean \pm SD (range) & $7.5 \pm 2.2(4.1-12.1)$ & $7.6 \pm 1.5(5.0-11.0)$ \\
\hline$\%$ male & 67 & 50 \\
\hline \% "Mother" listed as primary caregiver & 78 & 98 \\
\hline \% "Father" present in household & 62 & 90 \\
\hline$\%$ with reported ADHD & 76 & 0.2 \\
\hline $\begin{array}{l}\text { Hollingshead SES category, mean } \\
\text { I (highest; 55-66) n (\%) } \\
\text { II (40-54) } \\
\text { III (30-39) } \\
\text { IV (20-29) } \\
\text { V (lowest; 8-19) }\end{array}$ & $\begin{array}{l}\text { III } \\
8(24) \\
7(21) \\
10(30) \\
5(15) \\
3(9)\end{array}$ & $\begin{array}{c}\| \\
98(27.1) \\
162(44.7) \\
72(19.9) \\
26(7.2) \\
4(1.1)\end{array}$ \\
\hline$\%$ reported chronic medical problems ${ }^{b}$ & 18 & 8.5 \\
\hline
\end{tabular}

FASD, fetal alcohol spectrum disorders; SD, standard deviation; ADHD, attention deficit hyperactivity disorder; SES, socioeconomic status; aHollingshead SES data available for 362 of community sample subjects only; " most commonly reported problem in both groups was "asthma."

$\begin{array}{lcc}\text { Table 2-Representativeness of FASD sample } & & \\ & \text { FASD }(n=33) & \text { FAS DPN }(n=1054)^{\mathrm{a}} \\ \text { Age, years, mean } \pm \text { SD (range) } & 7.5 \pm 2.2(4.1-12.1) & 7.5 \pm 2.5(4.0-12.0) \\ \text { Male (\%) } & 67 & 59.4 \\ \text { Caucasian/White (\%) } & 60 & 48.0 \\ \text { CBCL Externalizing Problems with T-Score } \geq 67(\%)^{b} & 88 & 77 \\ \text { FAS/partial FAS (\%) } & 15 & 12 \\ \text { Static encephalopathy, alcohol exposed (\%) } & 21 & 31 \\ \text { Neurobehavioral disorder, alcohol exposed (\%) } & 64 & 57\end{array}$

FASD, fetal alcohol spectrum disorders; FAS DPN, Fetal Alcohol Syndrome Diagnostic and Prevention Network; CBCL, child behavior checklist; SD, standard deviation; ${ }^{\text {BBased on }} 1729$ patients enrolled in the WA state FAS Diagnostic and Prevention Network from 1993-2005 in target age range; b $\mathrm{A} C B C L$ score of $67-70$ is considered borderline, and $>70$ clinically significant.

This was an ethnically diverse sample, with $61 \%$ reported as Caucasian/White, 9\% African American, 6\% Hispanic, 6\% Native American, and 18\% reported to be "mixed race." Two participants had diagnoses consistent with the full FAS (6\%), 3 with partial FAS (9\%), 7 with "static encephalopathy/alcoholexposed" (21\%), and the remaining 64\% with "neurobehavioral disorder/alcohol-exposed." When compared to the community sample, the group with FASD was of similar age but had more males, less paternal presence in the household, and lower mean SES (Table 1).

\section{Caregiver Report of ADHD}

The study group with FASD had higher rates of caregiverreported ADHD when compared with the community sample, in which children who were taking psychostimulant medications (presumably for ADHD) were excluded for purposes of the original CSHQ validation study design (Table 1). ${ }^{21}$

\section{Representativeness of FASD Group}

The current study's FASD group is broadly representative of those found in the FAS DPN clinical database, which at the time of study enrollment included 1729 patients enrolled from 1993-2005. ${ }^{25,26}$ Sixty-one percent of the FAS DPN patients fell into the 4- to 12-year age range. Demographic, behavioral, and diagnostic characteristics of both the study and larger FAS DPN groups are similar, indicating that the current study cohort is reasonably representative of the larger WA State FASD database (Table 2).

\section{Subjective Sleep Assessment}

\section{CSHQ}

Caregivers of children with FASD reported higher levels of sleep complaints, with a mean Total Score of $51.7 \pm 11.0$ compared with $38.8 \pm 5.6$ among the community sample $(\mathrm{p}<0.001)$ (Table 3). This pattern was consistent across all 8 subdomain scores, though the differences in sleep disordered breathing were smaller with more variability in scores. In addition, $85 \%$ of children with FASD attained a Total Score above the clinical cutoff of 41 for sleep dysfunction compared with only $30 \%$ of the community sample $(p<0.001)$. While age, gender, SES, family structure, and presence of ADHD and presence of other chronic medical conditions were found to vary by participant group, none of these factors were found to correlate with CSHQ Total Score (data not shown). Despite an average of an hour less sleep at night in the FASD group (Table 3), both groups showed age-appropriate absence of habitual daytime napping (data not shown). 


\begin{tabular}{|c|c|c|c|}
\hline CSHQ & FASD $(n=33)$ & Community $(n=418)$ & $\mathrm{p}$ value \\
\hline Total Sleep Disturbance Score & $51.7 \pm 11.0$ & $38.8 \pm 5.6$ & $<0.001$ \\
\hline Percentage above clinical cutoff score $(\geq 41)$ & $85 \%$ & $35 \%$ & $<0.001$ \\
\hline \multicolumn{4}{|l|}{ Subdomain scores } \\
\hline Bedtime Resistance & $9.2 \pm 3.3$ & $7.1 \pm 1.9$ & $<0.001$ \\
\hline Sleep Onset Delay & $1.9 \pm 0.9$ & $1.3 \pm 0.5$ & $<0.001$ \\
\hline Sleep Duration & $4.7 \pm 2.1$ & $3.4 \pm 0.9$ & 0.001 \\
\hline Sleep Anxiety & $7.0 \pm 2.6$ & $4.9 \pm 1.5$ & $<0.001$ \\
\hline Night Wakings & $4.9 \pm 1.9$ & $3.5 \pm 0.9$ & $<0.001$ \\
\hline Parasomnias & $10.3 \pm 2.8$ & $8.1 \pm 1.3$ & $<0.001$ \\
\hline Sleep Disordered Breathing & $3.8 \pm 1.4$ & $3.3 \pm 0.7$ & 0.070 \\
\hline Daytime Sleepiness & $11.2 \pm 4.2$ & $9.6 \pm 2.8$ & 0.041 \\
\hline Usual duration of nighttime sleep (h) & $9.2 \pm 1.5$ & $10.5 \pm 0.6$ & $<0.001$ \\
\hline
\end{tabular}

FASD, fetal alcohol spectrum disorders.

Table 4-CSHQ findings by ADHD category a among children with FASD

CSHQ
Age in years
Male $(\%)$
Total Sleep Disturbance Score
Percentage above clinical cutoff score $(\geq 41)$
Subdomain scores
Bedtime Resistance
Sleep Onset Delay
Sleep Duration
Sleep Anxiety
Night Wakings
Parasomnias
Sleep Disordered Breathing
Daytime Sleepiness
Usual duration of nighttime sleep $(h)$

$\begin{aligned} & \text { FASD }(\mathrm{n}=33 \\ & 7.5 \pm 2.2 \\ & 67 \\ & 51.7 \pm 11.0 \\ & 85 \% \\ & 9.2 \pm 3.3 \\ & 1.9 \pm 0.9 \\ & 4.7 \pm 2.1 \\ & 7.0 \pm 2.6 \\ & 4.9 \pm 1.9 \\ & 10.3 \pm 2.8 \\ & 3.8 \pm 1.4 \\ & 11.2 \pm 4.2 \\ & 9.2 \pm 1.5\end{aligned}$

No AD/HD $(\mathrm{n}=7)$
$7.2 \pm 2.7$

71

$49.0 \pm 13.2$

$57 \%$

$9.3 \pm 3.8$

$1.4 \pm 0.8$

$3.7 \pm 1.1$

$6.7 \pm 2.3$

$5.0 \pm 2.2$

$10.9 \pm 3.3$

$3.0 \pm 0.0$

$10.4 \pm 4.9$

$10.0 \pm 0.8$

\section{AD/HD ( $\mathrm{n}=25)$ \\ $7.7 \pm 2.0$}

68

$51.9 \pm 10.4$

$92 \%$

$9.1 \pm 3.2$

$2.0 \pm 0.9$

$4.9 \pm 2.2$

$6.8 \pm 2.5$

$4.7 \pm 1.7$

$9.9 \pm 2.5$

$4.0 \pm 1.5$

$11.6 \pm 4.2$

$9.1 \pm 1.7$

CSHQ, Children's Sleep Habits Questionnaire; ADHD, attention deficit hyperactivity disorder; FASD, fetal alcohol spectrum disorder; a'Excludes one participant with FASD whose caregiver reported "unknown" on ADHD status.

Among those with FASD, CSHQ Total Scores did not vary greatly by presence or absence of ADHD, ascertained by parental report (Table 4). However, children with comorbid ADHD scored slightly higher on the following subscales: Sleep Duration, Sleep Disordered Breathing, and Daytime Sleepiness. A notably higher percentage of those with comorbid ADHD scored above the cutoff score of 41 . In addition, caregivers of children with ADHD reported nearly an hour less sleep per night than those without ADHD.

\section{Correlation of CSHQ Scores with FASD Severity}

All participants with full FAS or partial FAS $(n=5)$ attained CSHQ Total Scores above the clinical cutoff of 41 . In this relatively small sample, no associations emerged between the CSHQ Total Score and components of FASD diagnosis based on the 4-Digit Code reflecting: (1) growth deficiency (2) facial dysmorphology; (3) central nervous system dysfunction; and (4) level of prenatal alcohol exposure (data not shown).

\section{Objective Sleep Assessment (Polysomnography)}

All 28 children with FASD who had an elevated CSHQ Total Score were referred for a sleep medicine consultation. Ultimately, a sleep medicine evaluation and PSG were completed by 5 children (18\%) with an elevated CSHQ Total Score (range: 46-72) (Table 5). Given the small sample size, these data are considered exploratory.

Median age of the 5 children with FASD at time of PSG assessment was 7.5 years (IQR: 6.3, 7.5), and 60\% were male (Table 5). All 5 children had a caregiver-reported diagnosis of ADHD. There was a $14.3 \pm 8.6$ month mean time lapse between CSHQ and PSG, as sleep medicine consultations and PSG occurred based on family and clinical availability. All children tolerated PSG set-up and acquisition.

\section{Sleep Architecture}

There were no overt group differences between the 5 children and published normative values in sleep stage distribution, although ranges varied widely. Fragmented sleep, defined as 
Table 5-Polysomnography (PSG) findings

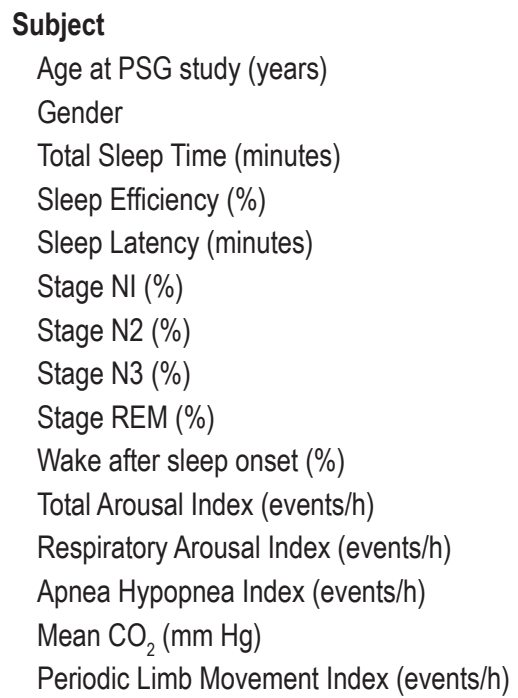

$\begin{array}{cc}\text { Summary Median; IQR } & \begin{array}{c}\text { Referent PSG }(\mathbf{n}=388)^{\mathrm{a}} \\ \text { Mean } \pm \text { SD }\end{array} \\ 7.5 ; 6.3,7.5 & 6.8 \pm 0.5 \\ 60.0 \% \text { male } & 51.5 \% \text { male } \\ 468.0 ; 430.0,514.0 & 471.6 \pm 43.7 \\ 90.0 ; 77.0,95.0 & 89.3 \pm 7.5 \\ 14.0 ; 13.0,32.0 & 23.0 \pm 25.3 \\ 5.2 ; 5.0,7.0 & 5.0 \pm 2.9 \\ 32.0 ; 29.0,37.0 & 41.8 \pm 8.3 \\ 34.0 ; 32.0,35.0 & 22.6^{\mathrm{b}} \\ 17.0 ; 16.9,24.0 & 19.6 \pm 4.3 \\ 5.0 ; 3.0,18.0 & 8.1 \pm 7.1 \\ 11.1 ; 10.0 .16 .0 & 9.3 \pm 4.8 \\ 2.0 ; 1.4,3.7 & 0.9 \pm 2.0 \\ 2.8 ; 2.2,4.0 & 0.7 \pm 0.8 \\ 45.0 ; 43.0,47.0 & 40.7 \pm 4.5 \\ 1.5 ; 0.0,1.6 & 1.0 \pm 1.4\end{array}$

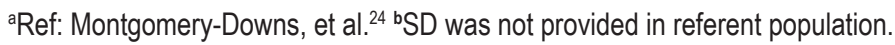

an elevated total arousal index, was seen among the 5 children with FASD. Median total arousal index was 11.1/h (IQR: 10, 16), and all children with FASD had higher than control group mean values. Respiratory arousal index correlated directly with severity of respiratory disturbances as measured by AHI (estimated slope $=0.7495 \%$ confidence interval (CI): 0.6-0.9). Total arousal index was only moderately associated with AHI (estimated slope $=0.5,95 \%$ CI: -1.1 to 2.1 ). Thus, not all arousals were directly associated with respiratory disturbances.

\section{Respiratory Events}

The median overall AHI was 2.8/h (IQR: 2.2, 4.0), higher than $2 \mathrm{SD}$ above the reported mean AHI in control data. Elevation of AHI in the children with FASD primarily arose from obstructive apneic and hypopneic events, and not central events. All participants with FASD had very mild snoring during PSG. Mean end-tidal $\mathrm{CO}_{2}$ levels were $45.2 \pm 3.5 \mathrm{~mm} \mathrm{Hg}$, higher than the reported control group mean of $40.7 \pm 4.5 \mathrm{~mm} \mathrm{Hg}$. Similar to sleep architecture measures, respiratory parameters showed a wide range of distributions.

\section{Periodic Limb Movements}

Periodic limb movements occurred with similar frequency to published control data.

\section{Seizures}

No clinical or electroencephalographic seizures were identified among the children with FASD.

\section{Medication Use}

Of the 5 children with FASD who underwent PSG, 3 were receiving a single medication and one was receiving multiple medications at the time of study; only one child was medication-free. A medication "washout" was refused by all the caregivers for this clinical evaluation. Families were concerned about the impact of a medication washout on child and family function. Medications were prescribed by non-sleep medicine specialists with the aims of decreasing daytime hyperactivity (amphetamine and non-amphetamine based stimulants), stabilizing mood (valproic acid, buspirone), and/or assisting with sleep initiation/maintenance (clonidine, melatonin) per caregiver report. All medications used are known to affect sleep architecture but not thought to significantly alter respiratory parameters during sleep. ${ }^{27}$

\section{DISCUSSION}

Overall prevalence of sleep abnormalities was high among children with FASD, with $\sim 85 \%$ scoring above a CSHQ cutoff for clinically significant sleep disorders. The elevation in average CSHQ Total Score for children with FASD was driven primarily by increased scores in subdomains related to the initiation and maintenance of sleep, best described as pediatric insomnia. Furthermore, within the FASD group, those with comorbid ADHD had further increased subdomain scores for Sleep Duration, Sleep Disordered Breathing, and Daytime Sleepiness compared with those without caregiver-reported ADHD, and they were also more likely to score above the Total Score threshold of 41 although mean Total Scores were not remarkably different. Exploratory PSG findings within a subset of these children with FASD aged 5 to 7 years, at a point in development when sleep should typically be at its most consolidated, demonstrate sleep disturbances best described as mild sleep disordered breathing primarily due to obstructive disturbances and hypoventilation, and fragmented sleep with slightly elevated arousal indices.

The results of the current study support prior clinical reports of elevated rates of erratically described "sleep problems" or "sleep disorders" among children with FASD in the range of $50 \%$ to $62 \%$ within heterogeneous clinical samples of children 
and adolescents diagnosed with FASD, ascertained using different diagnostic systems. ${ }^{1-3}$ In addition, this study augments the findings of a very recent systematic study focused on preschoolers, ${ }^{19}$ which also uses the validated CSHQ allowing us to directly compare results across groups. The current study expands the age range studied in FASD and adds exploratory data on objective polysomnography to characterize physiologic occurrences during what is reported to be very disturbed sleep in this population.

\section{Interpretation of CSHQ Results}

Reports of shortened sleep duration and frequent night wakings in children with FASD may be manifestations of SDB, other primary neurologic sequelae associated with in utero alcohol exposure, or comorbid neuropsychiatric disturbances. In this study, children with FASD scored higher on subdomains involving sleep onset (Bedtime Resistance, Sleep Onset Delay, Sleep Anxiety) and sleep maintenance (Sleep Duration, Night Wakings, Parasomnias). Except for the parasomnia category, this distribution of subjective complaints fits within a larger diagnostic category of pediatric insomnia, defined by a recent consensus statement as "repeated difficulty with sleep initiation, duration, consolidation, or quality that occurs despite age-appropriate time and opportunity for sleep and results in daytime functional impairment for the child and/or family." ${ }^{28}$ All these subjective complaints may contribute to complaints of daytime sleepiness, another elevated subdomain among children with FASD. Similar to findings in a younger cohort, ${ }^{19}$ the mean CSHQ Subdomain Score assessing SDB was not appreciably different than the control mean despite preliminary objective evidence from PSG indicating the presence of mild SDB in children with FASD. Caregiver observations are not the best predictors of obstructive sleep apnea ${ }^{29}$ which may account for this discrepancy. This supports the need for both subjective and objective data to best describe clinical sleep abnormalities at this juncture.

\section{Association with ADHD}

The clinical symptoms of FASD have some similarities to the diagnostic criteria for ADHD, resulting in likely diagnostic comorbidities. While exact prevalence rates of comorbid ADHD within the general population of FASD are unknown, the current study's caregiver reported rate of $76 \%$ falls within the ranges seen in other clinical samples, finding $60 \%$ to $95 \%$ of ADHD in persons with FASD. ${ }^{16}$ It is important to note that the attentional deficits seen with prenatal alcohol exposure may not have the same neuropsychological profile on detailed testing compared with non-teratogenic ADHD. ${ }^{30}$ Unfortunately, these tests are not readily available to most practitioners. Nevertheless, while FASD without symptoms of ADHD is not common, most pediatric practitioners who diagnose ADHD do not assess for any degree of prenatal alcohol exposure. Thus, within the population labeled as ADHD, the rate of FASD is unknown. In studies of children with ADHD, it is possible that some of the observed sleep problems arise through unascertained prenatal alcohol exposure. Therefore, clarifying diagnostic labels and the presence of prenatal alcohol exposure will be important in future studies of sleep problems in clinical samples of both ADHD and FASD.
While a comorbid diagnosis of ADHD in children with FASD may contribute to sleep problems in this population, it is unlikely to be the sole explanation. First, sleep problems are common in general pediatrics and not exclusive to those with ADHD or FASD. Indeed, $35 \%$ of subjects in the community sample had CSHQ scores $\geq 41$, despite the fact that those on psychostimulant medications (for presumed ADHD) were excluded from the community sample. ${ }^{21}$ Second, among the children with FASD in this study, those with and without ADHD still had higher Total Scores than controls, and higher than those with ADHD without known FASD. ${ }^{31}$ Meta-analyses of PSG findings in studies of children with a diagnosis of ADHD report both SDB and an elevation of periodic limb movements ${ }^{17}$; our pilot data in a subset of children with FASD and comorbid ADHD reveal SDB and hypoventilation, but no participant had an elevation in periodic limb movements. While this study was not designed to pinpoint the etiologies of differences between prior findings and this report's data, prenatal alcohol exposure is a potential culprit.

\section{Etiologic Possibilities for Sleep Problems in Children with FASD}

The preliminary PSG data can be used to explore the potential physiologic causes for subjective reports of pediatric insomnia and disrupted sleep in this clinical group, generating hypotheses to be evaluated using larger samples in studies that include carefully defined control groups.

One possible etiology is SDB, based on early evidence of elevated $\mathrm{AHI}$ and higher mean end-tidal $\mathrm{CO}_{2}$ measurements. Breathing irregularities in children with diagnosed FASD may have a physiologic basis. Experimental rodent models of prenatal alcohol exposure describe altered ventilatory responses. ${ }^{32}$ Children with FASD have known craniofacial abnormalities leading to narrowed upper airways, ${ }^{4}$ and reports show abnormal cerebellar vermi (thought to be involved with respiratory control and muscle coordination) $)^{33}$ in alcohol-exposed children. ${ }^{34}$ Any level of respiratory abnormalities may also result in repeated cortical arousals during sleep, compounding sleep fragmentation.

Another possible etiology for subjective complaints of insomnia lies in frequent cortical arousals leading to sleep fragmentation, presumably unrelated to SDB. This has previously been shown in other studies of prenatally alcohol-exposed infants and children and in animal experiments, with potential mechanisms including potential circadian and sleep homeostatic disturbances as a direct result of alcohol's teratogenic properties. ${ }^{35-40}$ The median number of cortical arousals in the current small sample undergoing PSG was high compared with controls when respiratory-related arousals were excluded. This suggests the increased occurrence of sleep fragmentation is not attributable only to SDB. It is possible, however, that some cortical arousals labeled as non-respiratory related were actually related to respiratory disturbances not detectable by current techniques.

\section{Limitations}

The current study is congruent with previous case series and a very recent systematic study of preschoolers in which high rates of concerning sleep problems were observed in children with FASD. Nevertheless, the data must be interpreted with 
potential study limitations in mind. First, the potential overlap between ADHD and FASD has been addressed but bears further study. Second, only children with clinically significant daytime behavior problems were enrolled into the original intervention study, from which participants in the current study were recruited. However, behavior problems are relatively common among children with FASD, and the children in this study were otherwise representative of the larger FAS DPN clinical database which had similar distribution of CBCL scores. Third, participants with FASD were not only exposed to alcohol before birth, but as a group also had other elevations in prenatal and postnatal psychosocial risk compared with controls, illustrated by lower SES scores and less traditional household/caregiver structures. However, there was still a high prevalence of reported sleep problems among those with FASD and low psychosocial risk (data not shown), indicating that psychosocial risk alone was not likely to have caused the difference between groups. This study was not designed to examine the role of these potential differences on CSHQ, but future studies with larger samples, including matched controls, may be able to do so. Fourth, comparison with the community sample, while quite informative, has limitations because these samples were recruited for different studies. It is unknown whether the community sample includes children with FASD. However, FASD prevalence data would suggest that only about four participants in the community sample would have fallen under the umbrella of FASD. Also, such misclassification of FASD cases as "controls" would have biased the comparison towards the null and away from the results that were actually found in this study. Lastly, the interpretation of PSG data was limited by very small numbers, referral and self-selection bias, and prescribed medications. However, data are in line with a recent study of medication-free preschoolers with FASD ${ }^{19}$ These pilot data illustrate the current state of clinical sleep medicine and difficulties in performing PSG in a group of children who are medicated for sleep problems. While these medications are prescribed without an evidence base, to discontinue medications for a study places caregivers and families at risk for recrudescence of worsened behaviors/sleep problems and their sequelae at home.

\section{Future Directions}

Though the results of the current study are based on a small sample and are preliminary, they cohere with data from existing basic science and clinical literature. The high prevalence of sleep problems observed in children with FASD suggests that children with FASD should routinely be evaluated for sleep complaints. Sleep problems in this heterogeneous group are likely impacted by multiple factors including the presence of comorbid ADHD, and screening for sleep problems should be done whether or not children show the classic characteristics of the full FAS, with referral to a sleep medicine specialist when appropriate. Sleep problems warrant assessment and treatment, possibly involving medications, as disturbed sleep may worsen the already compromised daytime behavior and adaptive function among children with FASD. An evidence base needs to be created upon which providers make decisions for treatment of sleep problems for those with FASD. Of special importance are study designs that include medication washout, if families can tolerate the functional impact of this procedure. Further elucidations of the mechanisms of alcohol's teratogenic effects on sleep are needed. Findings from this study highlight the urgency for clinical study of disrupted sleep among the relatively common but often underrecognized population of children with FASD.

\section{REFERENCES}

1. Steinhausen HC, Spohr HL. Long-term outcome of children with fetal alcohol syndrome: psychopathology, behavior, and intelligence. Alcohol Clin Exp Res 1998:22:334-8.

2. Bhatara $\mathrm{V}$, Loudenberg R, Ellis R. Association of attention deficit hyperactivity disorder and gestational alcohol exposure: an exploratory study. J Atten Disord 2006:9:515-22.

3. Green CR, Mihic AM, Nikkel SM, et al. Executive function deficits in children with fetal alcohol spectrum disorders (FASD) measured using the Cambridge Neuropsychological Tests Automated Battery (CANTAB). J Child Psychol Psychiatry 2009;50:688-97.

4. Astley SJ. Diagnostic Guide for Fetal Alcohol Spectrum Disorders. 3rd ed. Seattle, WA: University of Washington; 2004

5. Olson $\mathrm{HC}$, Ohlemiller MM, O'Connor MJ, et al. A call to action: Advancing essential services and research on fetal alcohol spectrum disorders. A report of the national task force on fetal alcohol syndrome and fetal alcohol effect. Atlanta, GA: Centers for Disease Control and Prevention; 2009.

6. May PA, Gossage JP, Kalberg WO, et al. Prevalence and epidemiologic characteristics of FASD from various research methods with an emphasis on recent in-school studies. Dev Disabil Res Rev 2009;15:176-92.

7. Kodituwakku PW. Defining the behavioral phenotype in children with fetal alcohol spectrum disorders: a review. Neurosci Biobehav Rev 2007;31:192-201.

8. Streissguth AP, Bookstein FL, Barr HM, et al. Risk factors for adverse life outcomes in fetal alcohol syndrome and fetal alcohol effects. J Dev Behav Pediatr 2004;25:228-38.

9. Olson HC, Oti R, Gelo J, et al. «Family matters:» Fetal alcohol spectrum disorders and the family. Dev Disabil Res Rev 2009;15:235-49.

10. Lupton $\mathrm{C}$, Burd L, Harwood R. Cost of fetal alcohol spectrum disorders. Am J Med Genet C Semin Med Genet 2004;127C:42-50.

11. Sadeh A, Gruber R, Raviv A. Sleep, neurobehavioral functioning, and behavior problems in school-age children. Child Dev 2002;73:405-17.

12. Capdevila OS, Kheirandish-Gozal L, Dayyat E, et al. Pediatric obstructive sleep apnea: complications, management, and long-term outcomes. Proc Am Thorac Soc 2008:5:274-82

13. Chervin RD, Ruzicka DL, Giordani BJ, et al. Sleep-disordered breathing, behavior, and cognition in children before and after adenotonsillectomy. Pediatrics 2006;117:e769-78.

14. Morgenthaler TI, Owens J, Alessi C, et al. Practice parameters for behavioral treatment of bedtime problems and night wakings in infants and young children: an American Academy of Sleep Medicine Report. Sleep 2006;29:1277-81.

15. Malow BA, Marzec ML, McGrew SG, et al. Characterizing sleep in children with autism spectrum disorders: a multidimensional approach. Sleep 2006;29:1563-71.

16. Rasmussen $\mathrm{C}$, Bena J, Pei J, et al. The impact of an ADHD co-morbidity on the diagnosis of FASD. Can J Clin Pharmacol 2010;17:e165-176.

17. Cortese S, Faraone SV, Konofal E, et al. Sleep in children with attention-deficit/ hyperactivity disorder: meta-analysis of subjective and objective studies. J Am Acad Child Adolesc Psychiatry 2009;48;894-908.

18. Walters AS, Mandelbaum DE, Lewin DS, et al. Dopaminergic therapy in children with restless legs/periodic limb movements in sleep and ADHD. Pediatr Neurol 2000;22:182-6

19. Wengel T, Hanlon-Dearman AC, Fjeldsted B. Sleep and sensory characteristics in young children with fetal alcohol spectrum disorder. J Dev Behav Pediatr 2011:32:384-92.

20. Tarasiuk A, Simon T, Tal A, et al. Adenotonsillectomy in children with obstructive sleep apnea syndrome reduces health care utilization. Pediatrics 2004;113:351-6.

21. Owens JA, Spirito A, McGuinn M. The Children's Sleep Habits Questionnaire (CSHQ): psychometric properties of a survey instrument for school-aged children. Sleep 2000;23:1043-51.

22. Hollingshead AB. Four factor index of social status. New Haven, CT: Yale University Press, 1975

23. Iber C, Ancoli-Israel S, Chesson AL Jr., et al. The AASM manual for the scoring of sleep and associate events. Rules, terminology and technical specifications. Westchester, IL: American Academy of Sleep Medicine; 2007. 
24. Montgomery-Downs HE, O'Brien LM, Gulliver TE, et al. Polysomnographic characteristics in normal preschool and early school-aged children. Pediatrics 2006;117:741-53.

25. Astley S. Washington State Diagnostic and Prevention Network: Summary of the first 1729 patient diagnosed from 1993 through 2005 (using the Diagnostic Guide for FASD: the 4-Digit Diagnostic Code, $3^{\text {rd }}$ Edition (Astley, 2004) [FAS DPN website]. 2009. Available at: http://depts.washington.edu/fasdpn. Accessed February 15, 2011.

26. Astley SJ. Profile of the first 1,400 patient receiving diagnostic evaluations for fetal alcohol spectrum disorder at the Washington state fetal alcohol syndrome diagnostic and prevention network. Can J Clin Pharmacol 2010;17:e132-e164.

27. Pelayo R, Dubik M. Pediatric sleep pharmacology. Semin Pediatr Neurol 2008:15:79-90.

28. Mindell JA, Emslie G, Blumer J, et al. Pharmacologic management of insomnia in children and adolescents: consensus statement. Pediatrics 2006;117:e1223-32.

29. Carroll J, McColley S, Marcus C, et al. Inability of clinical history to distinguish primary snoring from obstructive sleep apnea syndrome in children. Chest 1995;108:610-8.

30. O'Malley KD, Nanson J. Clinical implications of a link between fetal alcohol spectrum disorder and attention-deficit hyperactivity disorder. Can J Psychiatry 2002;47:349-54.

31. Owens JA, Maxim R, Nobile C, McGuinn M, Msall M. Parental and self-report of sleep in children with attention-deficithyperactivity disorder. Arch Pediatr Adolesc Med 2000;154:549-55.

32. Dubois $\mathrm{C}$, Houchi $\mathrm{H}$, Naassila $\mathrm{M}$, et al. Blunted response to low oxygen of rat respiratory network after perinatal ethanol exposure: involvement of inhibitory control. J Physiol 2008;586:1413-27.

33. Chen ML, Witmans MB, Tablizo MA, et al. Disordered respiratory control in children with partial cerebellar resections. Pediatr Pulmonol 2005;40:88-91.

34. Spadoni AD, McGee CL, Fryer SL, et al. Neuroimaging and fetal alcohol spectrum disorders. Neurosci Biobehav Rev 2007:31:239-45.

35. Hilakivi L, Tuomisto L, Hilakivi I, et al. Effect of prenatal alcohol exposure on neonatal sleep-wake behaviour and adult alcohol consumption in the AA and ANA rat lines. Alcohol Alcohol 1987:22:231-40.

36. Farnell YZ, Allen GC, Nahm SS, et al. Neonatal alcohol exposure differentially alters clock gene oscillations within the suprachiasmatic nucleus, cerebellum, and liver of adult rats. Alcohol Clin Exp Res 2008;32:544-52.

37. Ioffe S, Chernick V. Development of the EEG between 30 and 40 weeks gestation in normal and alcohol-exposed infants. Dev Med Child Neurol 1988;30:797-807.
38. Scher MS, Richardson GA, Coble PA, Day NL, Stoffer DS. The effects of prenatal alcohol and marijuana exposure: disturbances in neonatal sleep cycling and arousal. Pediatr Res 1988;24:101-5.

39. Troese M, Fukumizu M, Sallinen BJ, et al. Sleep fragmentation and evidence for sleep debt in alcohol-exposed infants. Early Hum Dev 2008;84:577-85.

40. Pesonen AK, Raikkonen K, Matthews K, et al. Prenatal origins of poor sleep in children. Sleep 2009;32:1086-92.

\section{ACKNOWLEDGMENTS}

The authors express deep appreciation to the participating families and dedicated clinical and research team of the Washington State Fetal Alcohol Syndrome Diagnostic and Prevention Network (FAS DPN), led by Susan J. Astley, Ph.D. The FAS DPN is the source of participants for this research project. Without the generous participation of families, and the unwavering efforts of the team to better serve individuals with FASD, this research would not have been possible. Work related to this study was performed at Seattle Children's Hospital and Seattle Children's Research Institute. Support for this study provided by Seattle Children's Research Institute Center for Clinical and Translational Research Mentored Scholar Program; U.S. Centers for Disease Control and Prevention, Grant number U01-0000DD038-05; and Seattle Children's Research Institute.

\section{SUBMISSION \& CORRESPONDENCE INFORMATION}

Submitted for publication August, 2011

Submitted in final revised form March, 2012

Accepted for publication March, 2012

Address correspondence to: Maida Lynn Chen, M.D., Seattle Children's Hospital, 4800 Sand Point Way, NE, MS A-5937, Seattle, WA 98105; Tel: (206) 987-1861; Fax:

(206) 987-2639; E-mail: maida.chen@seattlechildrens.org

\section{DISCLOSURE STATEMENT}

This was not an industry supported study. The author has indicated no financial conflicts of interest. 